\title{
A Design Evaluation of a User Interface for Tending Long-term Tasks
}

Robert Farrell

IBM T J Watson Research Center

19 Skyline Drive

Hawthorne, NY USA

robfarr@us.ibm.com

Hina Shah

Georgia Institute of Technology

266 Ferst Drive

Atlanta, GA 30332-0765

hinashah@cc.gatech.edu

Thomas Erickson

IBM T J Watson Research Center

Hawthorne, NY USA

snowfall@us.ibm.com

\section{Wendy A. Kellogg}

IBM T J Watson Research Center

19 Skyline Drive

Hawthorne, NY USA

wkellogg@us.ibm.com

\begin{abstract}
Organizational processes often take place over long periods of time and require intermittent attention. Remembering and reasoning about upcoming process tasks is important, but not adequately supported by existing tools. This paper describes Longitude, a tool that provides a compact timeline of tasks and deadlines. We discuss findings from an exploratory study of the system and propose new requirements for tools that help people participate in long-running group processes requiring intermittent and sporadic attention.
\end{abstract}

\section{Keywords}

Processes, organizations, to-do lists, task management

\section{ACM Classification Keywords}

H5.m. Information interfaces and presentation (e.g., HCI):

Miscellaneous.

\section{INTRODUCTION}

We are interested in designing tools that enable people to efficiently participate in group processes. Group processes in organizational settings often operate over long periods (e.g., a year), require action by many people, and involve a time-constrained sequence of intermittent tasks. Examples include performance reviews, skills assessment, and compliance training. 
Organizational processes are typically supported by a mix of human administrators and digital systems. Participants may become overloaded by automated notifications, messages from administrators, and reminders from management. This overload is exacerbated by the fact that people in large institutions often participate in multiple processes with differing time constraints. The premise behind this work is that it would be valuable to provide individuals with a unified view of all the processes in which they are enmeshed, and a means to remember tasks, anticipate dates, sequence work, and plan ahead.

\section{BACKGROUND}

HCI has had a longstanding interest in the support of group processes. Mostly this has involved workflow systems [e.g., [4], [7], but more recently researchers have explored the use of visualizations [e.g., [1], [5]]. In general, this work has taken an organizational perspective, focusing on systems for supporting the processes, and the impacts of that support.

In contrast, the perspective of the end user - the one on the receiving end of all the notifications, reminders and interrupts - has received less attention. Or rather, what has received attention is the more general problem of information overload (e.g., [9], [11]). A number of solutions, such as email-based task management systems (e.g., [2], [8]), have been proposed. However, little work has focused on end user management of organization-wide process tasks.

Organization-wide processes are a promising area for several reasons. First, they have special characteristics: they have many users, last for long periods of time, are cyclic, and the activity they require is intermittent.
Second, all organizations have processes, and these processes are becoming increasingly embedded in information systems. Third, they are a good point of leverage because organizations - unlike individuals have an incentive to moderate their behavior.

Because of the long-term, scheduled nature of organizational processes, we explored the use of timeline visualization. Timelines are, of course, a common means of displaying temporal data (e.g., [6], [10]). Our primary design goal was to provide a visual chronology of multiple processes over the span of approximately a year. We wanted to provide users with a timeline of the processes related to their position in the organization and to provide a shared visual context for better communication and coordination.

In $\mathrm{HCI}$, the canonical approach to system development is to begin with user studies, iteratively design prototypes with users, and then develop and deploy a system and study its use in situ. However, while we prefer this approach, in this case we 'inherited' the project, along with an interested executive sponsor and funding: a rare commodity in a large enterprise. The executive quickly provided a deadline for a working prototype. Despite the limited data available, we decided to proceed with the project as laid out, reasoning that a working prototype would provide a powerful means of gaining a wide range of experience.

\section{LONGITUDE: A TIMELINE VISUALIZATION}

Longitude is a Web-based graphical desktop widget

(Figure 1) that lays out graphical and textual entries on a timeline. The default view (shown) provides a compact 14-month overview. The icon and label for each entry are placed horizontally in time and stacked 
vertically to avoid text overlap. Clicking on a label pops up a window with a description, its date, and links to tools and resources. The user interface was created using the MIT SIMILE toolkit [3].

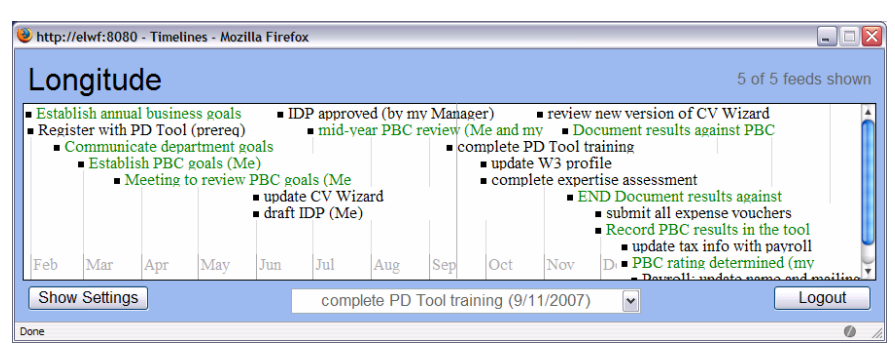

Figure 1: A timeline visualization of five processes

Process tasks are entered as to-do entries and a data feed of processes is created for each departmental group at various levels of an organization. After login, the widget accesses the user's department information and ascends the management hierarchy, subscribing to feeds. The user can then select one or more feeds to display and assign colors to distinguish, for example, different categories or priorities.

We have now deployed the prototype to an early adopter community for several months. At the time of publication, the system had over 500 registered users. However, system logs and user feedback revealed a low level of usage. We thus decided to investigate the strategies and tools currently used by people engaged in a large number of organizational processes to see if we could discover opportunities to improve on current practice.

\section{STUDY DESIGN AND METHOD}

We asked 31 leaders of professional groups at IBM to participate in our study and eight agreed. These professional groups consisted of employees in our research division sharing a common technical interest (e.g., "Multimedia"). The leaders were responsible for setting direction, managing activities, tracking expenses, and reporting to management. We also interviewed the original architect of the processes followed by all of the professional group leaders and the person providing administrative support.

Each session started with a semi-structured interview exploring participants' strategies and tools, followed by a walkthrough of Longitude. Before the session we asked the participants for one ongoing process associated with their professional group. We pre-loaded this process into the tool along with several other organizational processes. After logging in, participants were asked to describe what they saw and then start exploring; they were reminded to provide a verbal report of their thoughts as necessary. If they ran into problems, we helped them along. At the end of the session, we collected samples of artifacts, paper-based and digital, that they used to manage their tasks. Afterward, we exchanged email with participants to clarify any ambiguities emerging from our analysis of the collected artifacts.

All sessions were conducted by at least two people, one who guided the participant through the session, and the other(s) taking notes and managing recording. We transcribed seven of the recordings; two others were inaudible and a third was lost due to a technical problem. Our analysis involved summarizing each 
interview and then working through them several times, iteratively identifying a set of themes.

\section{RESULTS AND DISCUSSSION}

Participants reported spending between one and ten hours per week (more near deadlines) on tasks related to leading their professional group. Despite the ubiquity of a corporate to-do application, all eight participants instead maintained their to-do list(s) on paper, whiteboard, and/or text editors (Notepad $®$, emacs, Microsoft Word $($ ) $)$. All participants reported using a calendar to schedule meetings, but not for task management.

In the next section, we analyze the task management artifacts from four participants (P1 through P4). We then review the reactions of the study participants to the timeline.

\section{TASK MANAGEMENT ARTIFACTS}

P1 kept a single text file per month and had separate categories for 'urgent tasks', 'broad goals', 'detailed goals', and 'ideas'. Urgent tasks were very detailed such as 'send a note to Bob about approval for ACM sponsorship money' whereas many broader goals were less specific such as 'Bugs' and 'book travel'.

P2 maintained 4 text files per year. Her main "to-do" list had 1 line of text per task (e.g., "schedule meeting with Sam and Wendy"), each with a priority (0 to 9). At least once a week, she adjusted priorities, moved abandoned to a 'dropped' file, marked tasks as complete, and moved tasks to her 'done' file with a timestamp. Her 'laundry' file contained "things that I may want to look at one day" and entries were unstructured, but some were roughly categorized by affixing a prefix.
P3 planned participation in conferences using a timeline drawn on paper. The drawing covered a year, much like the Longitude visualization. However, there were several notable differences. First, the orientation of the timeline was vertical, allowing for room to write horizontally next to each deadline. Second, the timeline had notations for goals (circled), decisions (cross outs), commitments

(arrow from project to conference), and uncertain information that can later be filled in ('?' denotes unknown location). Third, it provided a compact, simple, uncluttered visualization by focusing on a single category of processes ("conferences"). Lastly, it offered a way of tracking and recording completion (shading).

P4 organized most of his tasks using a commercial notetaking application. He jotted down notes "in a quick manner" then periodically organized these into categories (e.g., company business processes, interest group activities, personal).

\section{REACTIONS TO THE TIMELINE VISUALIZATION}

Some of the participants' reactions to the timeline visualization were unexpected. What follows are some of the common reactions along with representative comments.

Longitude's timeline includes all tasks associated with a given process. Several participants were concerned about being overloaded by the number of tasks displayed. For example, P1 said "there would be so much on each month that I won't be able to see any other important stuff that's coming up". This reaction was partially because participants understood the timeline as a way to visualize all tasks, not just organizational process tasks. Several participants wanted to see a 
single process or one self-defined category of processes at a time.

Longitude was designed to visualize tasks taking place over long periods of time (e.g., a year), but participants reported that they "don't have a lot of time for looking far ahead" and are "not really concerned about these things that are so long term." Instead, most participants focused on what they needed to do "today or very soon". They wanted tasks to be "out of mind" until close to the deadline. They relied on colleagues, automated e-mails, or e-mails from administrators to remind them. Several participants reported deferring tasks until close to the deadline. One said, "I forget them [tasks] or delay them ... till it becomes a high priority". Two participants said they often postponed tasks until the deadline had actually arrived. Others pointed out the utility of deferring tasks that may not be well defined until the deadline approaches: "You can't really prepare too much because you never know how it develops."

Participants in our study used asterisks, circles, numbers, and other marks to notate individual task priority, changed the order of tasks to queue their execution, and scratched out (or removed) completed (or dropped) tasks. They also maintained various categories of tasks. For example, P1 had categories for urgent tasks, tasks to complete on the current day, next week's tasks, and categories for mostly longer-term tasks based on roles and projects. Future tasks were organized on an ad-hoc as-needed basis and longer-term tasks (e.g., "write patents") were typically not categorized. Thus, the work of categorizing and prioritizing was itself deferred until close to a deadline.
The timeline was designed for processes with specific tasks with fixed deadlines. However, far off tasks and broad goals tend to have a high degree of uncertainty. P3 noted that some things "don't have precise dates". He wanted to know where to place a task "that doesn't have a deadline." Participants also thought in terms of rough time periods. P3 said 'If I had a rough idea [for the date] I would put it on my paper calendar.' P2 had a 'laundry list' of possible things to do, and P1 had a list of rough

"ideas" that implied no commitment to accomplishment.

\section{DISCUSSION}

Given these results, we hypothesize that many of the task management practices and artifacts we observed can be seen as an attempt to reduce the inherent spatiotemporal and social uncertainty of organizational processes, that is, an attempt to deal with the 'laundry list' of vague goals, partial commitments, unscheduled tasks, and unclear possibilities that come with being part of a complex functioning organization.

Task management tools need to manipulate goals and tasks in varied degrees of specification, commitment, and scheduling, with support for rough categories and changing priorities, and accommodating a wide range of individual preferences. Tasks associated with deadlines could be gradually revealed as the deadlines approach. The primary view could be near-term, but provide ready access to other tasks in the same category or time frame to enable users to incrementally categorize, prioritize, or schedule tasks.

We anticipate that timeline visualization could be most useful in the context of calendaring, where long-term goals, milestones, and deadlines can be reviewed while planning out future activities. Participants in long- 
running processes will need a tool that supports asneeded, intermittent, and sporadic "tending" to longterm tasks. Meeting these requirements presents a new and exciting challenge for designers of task management systems.

\section{CONCLUSION AND FUTURE WORK}

Group processes are a common and often critical part of how organizations work, yet relatively little research has focused on tools to help end users manage their participation. We developed the Longitude timeline visualization tool to provide a unified long-term view of tasks and deadlines. During our interviews and walkthroughs we found that people prefer a short-term view of tasks, and defer planning, categorizing, and prioritizing tasks until close to a deadline. More research is needed to explore new designs for task management tools that better adapted to the sporadic and intermittent attention required by people engaged in complex processes.

\section{ACKNOWLEDGMENTS}

We thank Paul Moody who initiated the work on the timeline and the study participants for their time and input. Thanks also to Dave Newbold from the IBM CIO's office who provided early funding for the Longitude project.

\section{REFERENCES}

[1] Bellamy, R.K.E. et al, "Seeing is believing: Designing visualizations for managing risk and compliance" IBM Systems Journal, 46, 2 (2007), 205-218.

[2] Bellotti, V., Ducheneaut, N., Howard, M., and Smith, I. Taking email to task: the design and evaluation of a task management centered email tool. In Proc. $\mathrm{CHI}$ 2003. ACM Press (2003), 345-352

[3] Butler, M, H., Gilbert, J., Seaborne, A., Smathers, $\mathrm{K}$.. Data conversion, extraction and record linkage using $\mathrm{XML}$ and RDF tools in Project SIMILE. HP Labs Technical Report HPL-2004-147, 2004.

[4] Dourish, P. Process descriptions as organisational accounting devices: the dual use of workflow technologies. Proc. GROUP 2001. ACM Press (2001), 5260

[5] Erickson, T., Huang, W., Danis, C., and Kellogg, W.A. A social proxy for distributed tasks: Design and evaluation of a prototype. Proc. CHI 2004, ACM Press (2004), 559-566.

[6] Freeman, E. and Gelernter, D. Lifestreams: A Storage Model for Personal Data. SIGMOD Record, 25, 1 (1996).

[7] Grinter, R. E. Workflow Systems: Occasions for Success and Failure. Computer Supported Cooperative Work, 9, 2 (2000), 189-214.

[8] Gwizdka, J. TaskView: design and evaluation of a task-based email interface. In Proceedings of the 2002 Conference of the Centre For Advanced Studies on Collaborative Research (2002).

[9] González, V. M. and Mark, G. "Constant, constant, multi-tasking craziness": managing multiple working spheres. Proc. CHI 2004. ACM Press (2004), 113-120.

[10] Plaisant, C., Milash, B., Rose, A., Widoff, S., and Shneiderman, B. Lifelines: Visualizing Personal Histories. Proc. CHI 1996. ACM Press, (1996) 221-227.

[11] Whittaker, S. and Sidner, C. Email overload: exploring personal information management of email. In Proc. CHI 1996. ACM Press (1996), 276-283. 